\title{
BLOW UP OF NONAUTONOMOUS FRACTIONAL REACTION-DIFFUSION SYSTEMS
}

\author{
AROLDO PÉREZ
}

Abstract. We provide a sufficient condition for finite time blow up of the positive mild solution to the nonautonomous Cauchy problem of a reaction-diffusion system with distinct fractional diffusions. The proof is based on the reduction to an ordinary differential system by means of a comparison between the transition densities of the semigroups generated by the different fractional Laplacians. Moreover, we prove that this condition is also a sufficient condition for the blow up of a related nonautonomous fractional diffusion-convection-reaction system.

Mathematics subject classification (2010): 35B44, 35C15, 35K57, 35S10.

Keywords and phrases: Fractional Laplacian, non-linear reaction-diffusion systems, finite time blow up, mild solution.

\section{REFERENCES}

[1] J. BeBERnES AND D. EBERLy, Mathematical problems from combustion theory, Applied Mathematical Sciences 83, Springer-Verlag, New York, 1989.

[2] K. BOGDAN AND T. JAKUBOWSKI, Estimates of heat kernel of fractional Laplacian perturbed by gradient operators, Commun. Math. Phys. 271, (2007), 179-198.

[3] M. Escobedo And M. A. Herrero, Boundedness and blow up for a semilinear reaction-diffusion system, J. Differential Equations 89, (1991), 176-202.

[4] A. Z. FINO AND M. KIRANE, Qualitative properties of solutions to a time-space fractional evolution equation, Quart. Appl. Math. 70, 1 (2012), 133-157.

[5] H. FuJitA, On the blowing up of solutions of the Cauchy problem for $u_{t}=\Delta u+u^{1+\alpha}$, J. Fac. Sci. Univ. Tokyo Sect. I 13, (1966), 109-124.

[6] M. GUEDDA AND M. KIRANE, Criticality for some evolution equations, Differential Equations 37, 4 (2001), 511-520.

[7] T. KAKEHI AND Y. OSHITA, Blowup and global existence of a solution to a semilinear reactiondiffusion system with the fractional Laplacian, Math. J. Okayama Univ. 59, (2017), 175-218.

[8] M. KiRAnE AND M. QAFSAOUI, Global nonexistence for the Cauchy problem of some nonlinear reaction-diffusion-systems, J. Math. Anal. Appl. 268, (2002), 217-243.

[9] C. M. KirK And W. E. Olmstead, Superdiffusive blow-up with advection, Int. J. Dynamical Systems and Differential Equations 4, Nos. 1/2 (2012), 93-102.

[10] J. A. López-Mimbela And J. Villa-Morales, Local time and Tanaka formula for a multitype Dawson-Watanabe superprocess, Math. Nachr. 279, 15 (2006), 1695-1708.

[11] A. PÉrEZ, A blow up condition for a nonautonomous semilinear system, Elec. J. Diff. Equations 2006, 94 (2006), 1-8.

[12] A. PÉREZ, Blow up of fractional reaction-diffusion systems with and without convection terms, J. Integral Equations Applications 30, 1 (2018), 181-196.

[13] A. PÉRez And J. Villa, Blow-up for a system with time-dependent generators, Lat. Am. J. Probab. Math. Stat. 7, (2010), 207-215.

[14] A. A. Samarskit, V. A. Galaktionov, S. P. Kurdyumov and A. P. Mikhailov, Blow-up in quasilinear parabolic equations, de Gruyter Expositions in Mathematics 19, de Gruyter, Berlin, 1995.

[15] K. SATo, Lévy processes and infinitely divisible distributions, Cambridge Studies in Advanced Mathematics 68, Cambridge University Press, Cambridge, 1999. 
[16] M. F. Shlesinger, G. M. Zaslavsky And U. Frisch (EDS), Lévy fligths and related topics in Physics, Lecture Notes in Physics 450, Springer-Verlag, Berlin, 1995.

[17] S. Sugitani, Nonexistence of global solutions for some nonlinear integral equations, Osaka J. Math. 12, (1975), 45-51.

[18] A. S. TERSENOV, The preventive effect of the convection and of the diffusion in the blow-up phenomenon for parabolic equations, Ann. I. H. Poincaré-AN 21, (2004), 533-541.

[19] Y. UDA, The critical exponent for a weakly coupled system of the generalized Fujita type reactiondiffusion equations, Z. Angew. Math. Phys. 46, (1995), 366-383.

[20] J. Villa-Morales, Blow up of mild solutions of a system of partial differential equations with distinct fractional diffusions, Elec. J. Diff. Equations 2014, 41 (2014), 1-9.

[21] E. WU AND Y. TANG, Blow-up solutions to the Cauchy problem of a fractional reaction-diffusion system, J. Inequal. Appl. 2015, 123 (2015), 1-18. 